\title{
An efficient data collection path planning scheme for wireless sensor networks with mobile sinks
}

\author{
Jau-Yang Chang ${ }^{*}$, Jin-Tsong Jeng, Yung-Hoh Sheu, Z.-Jie Jian and Wei-Yeh Chang
}

*Correspondence:
jychang@nfu.edu.tw
Department of Computer
Science and Information
Engineering, National
Formosa University, Hu-Wei,
Yun-Lin, Taiwan

Yun-Lin, Taiwan

\begin{abstract}
Wireless sensor networks with mobile sinks enable a mobile device to move into the sensing area for the purpose of collecting the sensing data. Mobile sinks increase the flexibility and convenience of data gathering in such systems. Taking the energy consumption of the mobile sink into account, the moving distance of the mobile sink must be reduced efficiently. Hence, it is important and necessary to develop an efficient path planning scheme for mobile sinks in large-scale wireless sensor network systems. According to several greedy-based algorithms, we adopt an angle bisector concept to create the moving path for the mobile sink. In this paper, a novel and efficient data collection path planning scheme is proposed to reduce the moving distances and to prolong the lifetimes of mobile sinks in wireless sensor networks. Considering the communication range limitations of sensor nodes and the obstacles within sensing areas, we design an inner center path planning algorithm to reduce the moving distance for the mobile sink. A back-routing avoidance method is included to address the moving path backpropagation problem. We account for the obstacles in sensing area. The reference point of obstacle avoidance is employed to address the obstacle problem. The proposed scheme makes an adaptive decision for creating the moving path of the mobile sink. A suitable moving path planning scheme can be achieved, and the moving distance of the mobile sink can be reduced. The proposed scheme is promising in large-scale wireless sensor networks. When the number of sensor nodes in the sensing area is increased by 50, the proposed scheme yields an average moving distance that is $1.1 \mathrm{~km}$ shorter than that of the heuristic tour-planning algorithm, where the sensing area is $5 \mathrm{~km} \times 5 \mathrm{~km}$. Simulation results demonstrate that the proposed data collection path planning scheme outperforms the previously developed greedy-based scheme in terms of the moving paths and moving distances of mobile sinks in wireless sensor networks.
\end{abstract}

Keywords: Data collection, Mobile sink, Moving distance, Moving path, Sensor node, Wireless sensor networks

\section{Introduction}

Wireless sensor networks (WSNs) with mobile sinks have been widely considered a promising solution to replace people for discovering and collecting sensing data within a highly dangerous region [1-5]. The mobile sink can be an intelligent moving author(s) and the source, provide a link to the Creative Commons licence, and indicate if changes were made. The images or other third party material in this article are included in the article's Creative Commons licence, unless indicated otherwise in a credit line to the material. If material is not included in the article's Creative Commons licence and your intended use is not permitted by statutory regulation or exceeds the permitted use, you will need to obtain permission directly from the copyright holder. To view a copy of this licence, visit http:// creativecommons.org/licenses/by/4.0/. 
robot or unmanned aircraft that enters the sensing area for the purpose of collecting the sensing data. Figure 1 shows the environment of WSNs with mobile sinks. In large-scale WSNs, many sensor nodes are deployed in the sensing area. All of the sensor nodes have sensing, computing and wireless communication capabilities. Each sensor node plays the role of an event detector to monitor specific targets and sends the data to a sink or a base station (BS) by using wireless transmission techniques. It is intuitive that the sensor nodes need more transmission power to send data when a sink or a BS is fixed and located far from the sensor nodes than when it is close to the nodes. The energy efficiency of the sensor nodes is one of the most important features for extending the lifespans of WSNs. A mobile sink is employed to increase the flexibility of the network for collecting the sensing information in largescale monitoring environments. Because a sink can randomly move within the sensing area to gather the detection data, the data transmission distances of the sensor nodes can be reduced. The mobility of the sink enables convenient data collection and reduces the energy consumption by the sensor nodes [6-10]. WSNs with mobile sinks are expected to be used to collect sensing data in highly dangerous regions, including battlefields, disaster areas and environmental pollution areas. In most application scenarios, the main power supply of a mobile sink is its battery. Taking the energy consumption of the mobile sink into account, the moving distance of the mobile sink must be reduced efficiently. Hence, saving energy becomes one of the most important factors for a mobile sink to extend its lifetime. To reduce the moving distances and to prolong the lifetimes of mobile sinks, a new and efficient data gathering path planning scheme must be developed for large-scale WSN systems. Hence, the performance parameters of interest in this paper are the moving path and moving distance of a mobile sink.

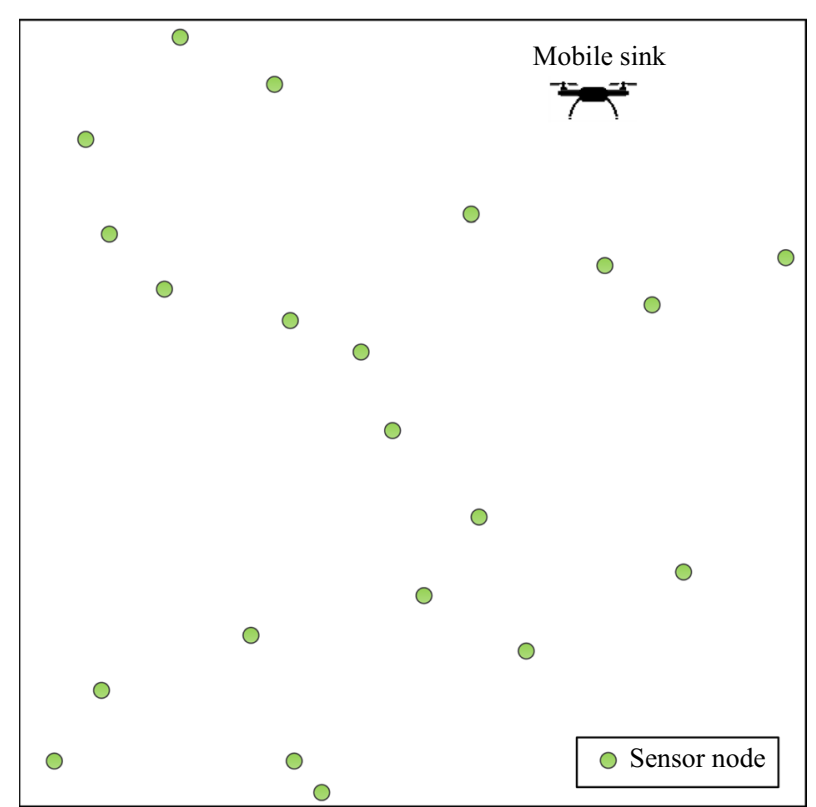

Fig. 1 Environment of a WSN with a mobile sink. The mobile sink can be an intelligent moving robot or unmanned aircraft that enters the sensing area for the purpose of collecting the sensing data 
The remainder of this paper is organized as follows. In Sect. 2, we review related studies from the literature. In Sect. 3, we describe the system model of a WSN with mobile sinks and illustrate the approach of the data gathering path planning scheme with an inner center in detail. In Sect. 4, a performance analysis is presented. We evaluate the performance of our proposed scheme by using a simulation model. Finally, conclusions are given in Sect. 5 .

\section{Related works}

Several studies on mobile sink issues have been conducted for WSNs. With regard to the energy saving schemes used for the sensor nodes, analytical models have been proposed in [3-8]. The main ideas of these schemes are dependent on the path taken by the mobile sink to reduce the energy consumption of sensor nodes. In [3], the authors determined the best location for a mobile sink according to the most efficient energy consumption by cluster heads ( $\mathrm{CHs}$ ) for data delivery after clustering the sensor nodes and selecting the CHs. In [4], an energy-aware sink relocation strategy for mobile sinks in WSNs was proposed to avoid consuming too much energy for a specific group of sensor nodes. To extend the lifetime of the WSN, this strategy uses information related to the residual energy of sensor nodes to adaptively adjust the transmission range of the sensor nodes and the relocation scheme for the sink. In [5], the authors proposed a weighted rendezvous planning scheme to form a hybrid moving pattern in which a mobile sink only visits rendezvous points, as opposed to all sensor nodes. Sensor nodes that are not rendezvous points transmit their sensing data to the nearest rendezvous point by using a multihop approach. In [6], the authors proposed a ring routing protocol for wireless sensor networks with mobile sinks to provide load-balanced data delivery and achieve uniform energy consumption across the network. The protocol is suitable for time-sensitive applications, which aim to minimize overhead in terms of energy consumption and packet delays. In [7], the authors proposed a mobile sink-based adaptive immune energy-efficient clustering protocol to find the optimal number of $\mathrm{CHs}$ and to improve the lifetime and stability period of the network. The protocol also guides the mobile sink with the objective of minimizing the total dissipated energy during communication and the amount of overhead control packets. In [8], a tree-based power saving scheme was proposed to reduce the energy consumption in wireless sensor networks with mobile sinks. The main goal of this scheme is to reduce the data transmission distances of the sensor nodes by employing a dynamic sorting algorithm to achieve tree-cluster routing using multihop concepts. In [9], the authors proposed an energy-efficient routing protocol based on particle swarm optimization and a genetic algorithm to prevent energy dissipation between sensor nodes; by calculating the load-balanced table of clusters, the optimal set of data gathering points and an optimal path for the mobile sink can be obtained. In [10], an energy-efficient intracluster routing algorithm was proposed to balance the energy consumption among member nodes by optimizing the sojourn locations of the mobile sink in each cluster.

With regard to the data collection methods for mobile sinks, analytical models have been proposed in [11-20]. In [11], an energy-aware path construction algorithm was proposed to prolong the lifetimes of WSNs with mobile sinks by selecting an appropriate set of data collection points, constructing a data collection path and collecting data from 
the points burdened with too much data. In [12], a mobile data gathering algorithm with multihop transmission was proposed to reduce the delay time of data gathering in WSNs by visiting the convergence area of sensors' communication ranges. In [13], the authors studied the compressive data gathering problem in terms of Bayesian theory for WSNs with mobile sinks. The main goal of this scheme is to reduce the latency of the network by manually selecting the schedule of gathering nodes. In [14], the authors investigated the problem of data collection with multiple sinks. To find the optimal sink location, they designed a suboptimal online algorithm via a primal-dual approach with little a priori knowledge. In [15], the authors presented analytical approaches to determine the energy consumption of nodes for large-scale wireless sensor networks by using mobile data collection schemes and providing models for determining the optimal number of clusters for minimizing the energy consumption. In [16], a hybrid routing scheme was proposed to reduce the energy consumption of the network. The authors determined the optimal movement trajectory for the mobile sink by using the Hilbert space-filling curve based on the change in the amounts of data generated in different network regions. In [17], the authors formulated the data gathering problem as a network utility maximization problem for rechargeable sensor networks; their aim was to maximize the total amount of data collected by the mobile sink while maintaining the fairness of the network. In [18], the authors proposed a comprehensive data gathering scheme based on a graphing technique to optimize the energy consumption of all sensor nodes in WSNs with mobile sinks. In [19], the authors proposed a three-phase, energy-balanced heuristic scheme to prolong the lifetime of the network. The network region is divided into grid cells that are assigned to clusters by using the $\mathrm{k}$-dimensional tree algorithm. The main goal of this approach is to ensure that the multiple mobile sinks have similar energy consumption levels for both data gathering and sink movement. In [20], the authors proposed a virtual grid-based dynamic route adjustment scheme to minimize the route reconstruction costs of the sensor nodes for mobile sink-based WSNs. While moving around the sensor field, a mobile sink continues to change its location and interacts with the $\mathrm{CH}$ node closest to the borderline for data collection.

Several studies on obstacle avoidance issues have been proposed in [21-28], and these studies have been validated to some extent through simulations. In [21], the authors presented an energy-efficient routing mechanism based on the clustering method for mobile sinks in WSNs with obstacles. A heuristic tour-planning algorithm (HTPA) was proposed to find the shortest route for the mobile sink while avoiding obstacles. In [22], the authors presented a dynamic movement approach that offers path planning with obstacle avoidance to minimize the localization error while maximizing the number of successful localized nodes. In [23], the authors presented a simultaneous trajectory planning and tracking controller for use under cruise conditions based on a predictive control model to address obstacle avoidance for an intelligent vehicle. In [24], a computationally efficient online local motion planning algorithm was proposed for mobile robots in unknown, cluttered dynamic environments. In [25], the authors presented an obstacle avoidance and path planning algorithm for a multi-joint manipulator in a space robot. The end effector of the manipulator is used to capture some special target in a space environment with obstacles. In [26], the authors focused on the dynamic obstacle avoidance and path planning problem of unmanned surface vehicles and attempted to 
solve it by constructing an automatic obstacle avoidance method based on the ant colony algorithm and the clustering algorithm. In [27], the authors proposed a hybrid algorithm that effectively combines global and local path planning. Global path planning involves generating a global path for an unmanned surface vehicle to enable it to reach the target point. Local path planning involves avoiding dynamic obstacles and tracking the global path by following the local target point, which is the intersection of the target points indicated by global and local path planning. In [28], the authors proposed an obstacle avoidance method based on the law of conservation of energy to adjust the motion states of robot manipulators in real time.

Several protocols for energy management have been implemented on WSNs. In [29], the authors proposed a variant of the low-energy adaptive clustering hierarchy (LEACH) algorithm called the partitioned-based energy-efficient LEACH (PE-LEACH) protocol, which tends toward the energy-based fault-tolerant technique. $\mathrm{LEACH}$ is a clusterbased routing protocol that uses a clustering approach [30]. In the sensing area, a large number of sensor nodes are divided into several clusters. For each cluster, a sensor node is selected as a $\mathrm{CH}$ based on a predetermined probability. Other non- $\mathrm{CH}$ nodes choose the nearest clusters to join by receiving the strength of the advertisement message from the $\mathrm{CH}$ nodes. Other cluster-based energy saving schemes for WSNs derived from the LEACH protocol have been proposed in [31-33], and they have been validated to some extent through simulations. The main difference between these schemes is the process for selecting a $\mathrm{CH}$ node. However, the critical problem is that the random selection of a $\mathrm{CH}$ node could result in a poor clustering setup.

With regard to the previously developed schemes described above, only a few research papers have studied the problem of the energy consumption of mobile sinks in WSNs. Additionally, the assumption of the communication range of sensor nodes must be practical and reasonable. Due to terrain constraints, it is clear that obstacles should be considered in the real environments of WSNs with mobile sinks. Motivated by the above discussion, we propose a novel energy-efficient data collection path planning scheme with an inner center to reduce the moving distances and to prolong the lifetimes of mobile sinks in wireless sensor networks. The main principles and contributions of our proposed scheme are summarized as follows:

- Considering the communication range limitations of sensor nodes and the obstacles within sensing areas, the proposed scheme makes an adaptive decision for creating the moving path of the mobile sink. A beneficial moving distance for the mobile sink is obtained. This implies that the energy consumption of the mobile sink is reduced and that the task of data collection for the mobile sink can be accelerated.

- Our proposed scheme has the ability to offer mobile devices an efficient data collection path planning method for WSNs with mobile sinks. In addition, our simulation system accounts for environments that are encountered in practice. Different numbers of sensor nodes and sensing areas with obstacles are simulated and discussed in this paper. This discussion is important for evaluating the performance of the moving path, moving distance and lifetime of a mobile sink.

- Because of the constraints with regard to simulating the communication range limitations of sensor nodes and the obstacles, the performance analysis is conducted via 


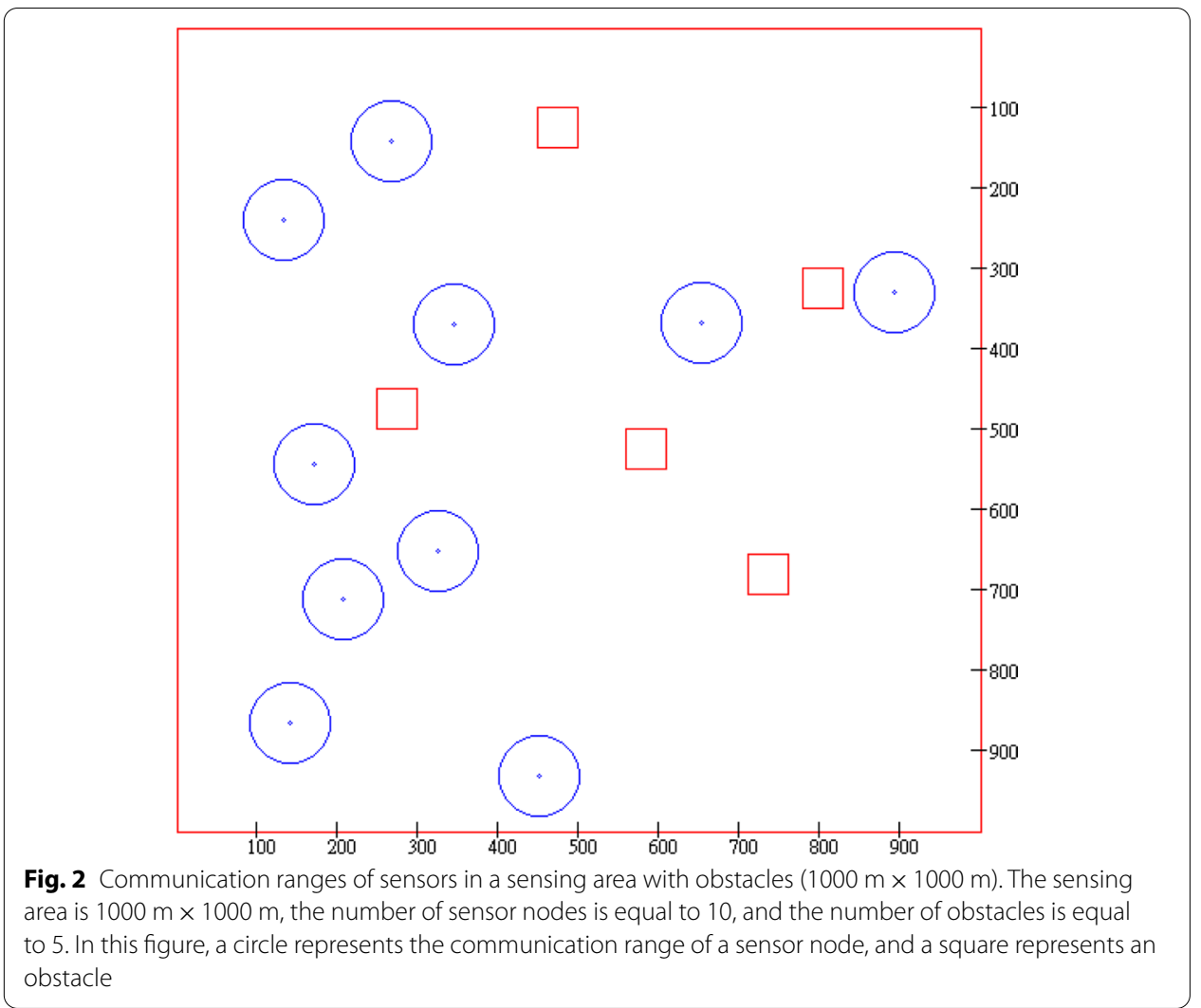

a connection-level simulation. This approach allows us to use a realistic model. In this paper, we design a visual interface simulator to discuss and analyze the moving path and moving distance of a mobile sink.

- The important feature of our proposed framework is that it addresses the moving path backpropagation problem faced by the greedy-based path planning algorithm. A reasonable moving path can be utilized, and a suitable moving distance for the mobile sink can be obtained in a WSN. The main benefit of our proposed scheme is that it shortens the moving distances of mobile sinks in WSNs.

\section{Methods}

\subsection{System model and problem description}

The system infrastructure is composed of a mobile sink and some fixed sensor nodes. The mobile sink can be an intelligent moving robot or unmanned aircraft that randomly moves within the sensing area for collecting the sensing data in an outdoor environment. The main power supply of a mobile sink is its battery, and the battery cannot be recharged. Hence, the mobile sink in the sensing area is energy-constrained. We assume that the energy of a sensor node can be recharged and that the transmission power of the sensor node is fixed. This implies that a stable communication range can be used for each sensor node. In this paper, we assume that the locations of the sensor nodes and obstacles are known and that the communication range of each sensor node is fixed. Figure 2 shows an example of the communication ranges of sensors in a sensing area 


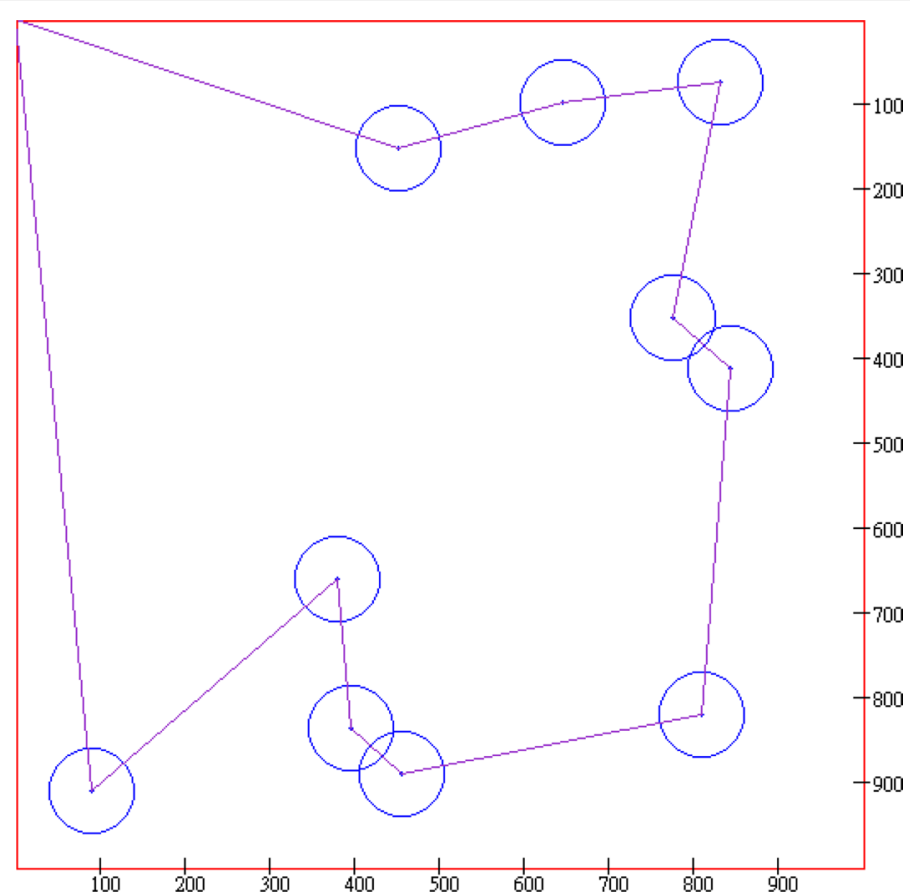

Fig. 3 Example of a moving path for the CCPPA (1000 m × $1000 \mathrm{~m})$. Example of a moving path for a certain case in the simulation by using the greedy-based center of circle path planning algorithm (CCPPA), where the top left corner is the starting position and there are no obstacles. The mobile sink travels through all of the sensor nodes and then moves back to the starting position

with obstacles, where the sensing area is $1000 \mathrm{~m} \times 1000 \mathrm{~m}$, the number of sensor nodes is equal to 10 , and the number of obstacles is equal to 5 . A circle represents the communication range of a sensor node, and a square represents an obstacle.

The greedy-based path planning algorithm is a shortest path prioritizing method, and it is illustrated step by step as follows.

Input: $\mathrm{M}(x, y)$ (the location of mobile sink), $\mathrm{S}(x, y)$ (the location of sensor node), $\mathrm{N}$ (the number of sensor nodes).

Output: The moving path of the mobile sink.

Initialization: $\mathrm{M}(x, y)=\mathrm{M}(0,0)$ (The initial location of the mobile sink), $k=1$ (The number of sensor node locations selected), and the temporary result $=$ null.

Step 1: The mobile sink calculates the distances between all $\mathrm{S}(x, y)$ and $\mathrm{M}(x, y)$.

Step 2: The minimal distance between $\mathrm{S}(x, y)$ and $\mathrm{M}(x, y)$ is selected.

Step 3: Let $\mathrm{M}(x, y)=\mathrm{S}(x, y)$ and remove $\mathrm{S}(x, y)$.

Step 4: Let $k=k+1$. If $k>\mathrm{N}$, go to Step 5. Otherwise, go to Step 1.

Step 5: The temporary result is the final moving path of the mobile sink, and the moving distance of the mobile sink can be calculated.

We design a visual interface simulator by using MATLAB and C\# to discuss and analyze the moving path and moving distance of the mobile sink. Figure 3 shows an example of a moving path for a certain case in the simulation by using the greedy-based center of circle path planning algorithm (CCPPA), where the top left corner is the starting position and there are no obstacles. The mobile sink travels through all of the sensor nodes and then moves back to the starting position. The greedy-based path planning algorithm 


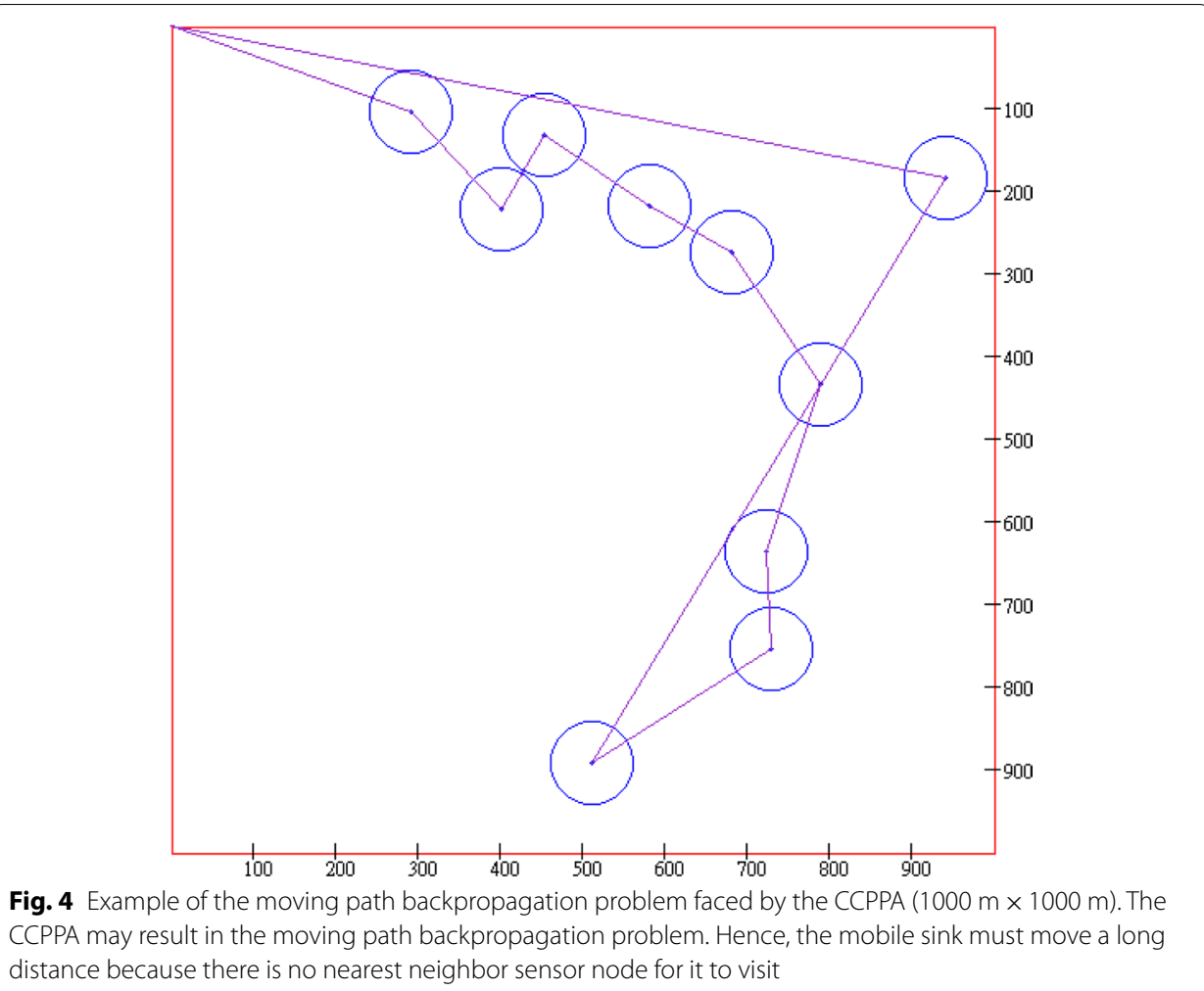

may result in the moving path backpropagation problem. Hence, the mobile sink must travel an increased distance because there is no nearest neighbor sensor node for it to visit. Figure 4 shows an example of the moving path backpropagation problem for a certain case in the simulation by using the CCPPA.

The design of the moving path is an important issue in WSNs with mobile sinks. It is obvious that the moving distance is responsible for most of the energy consumption by the mobile sink. Additionally, the moving path backpropagation problem leads to increased energy consumption by the mobile sink. Accounting for the energy consumption of the mobile sink, the moving distance must be reduced. Hence, the moving path backpropagation problem should be solved, and an efficient path planning scheme for mobile sinks in sensing areas with obstacles should be developed.

\subsection{Proposed moving path scheme}

Considering the communication range limitations of sensor nodes and the obstacles within sensing areas, we design an inner center path planning algorithm (ICPPA) to reduce the moving distance of the mobile sink. A back-routing avoidance method is included to address the moving path backpropagation problem. Based on the greedy path planning algorithm, Fig. 5 shows the flowchart of the back-routing avoidance method, where $M(x, y)$ is the location of the mobile sink, $M(0,0)$ is the starting position of the mobile sink, $L(x, y)$ are the locations of the sensor nodes, and $N$ is the number of sensor nodes. 


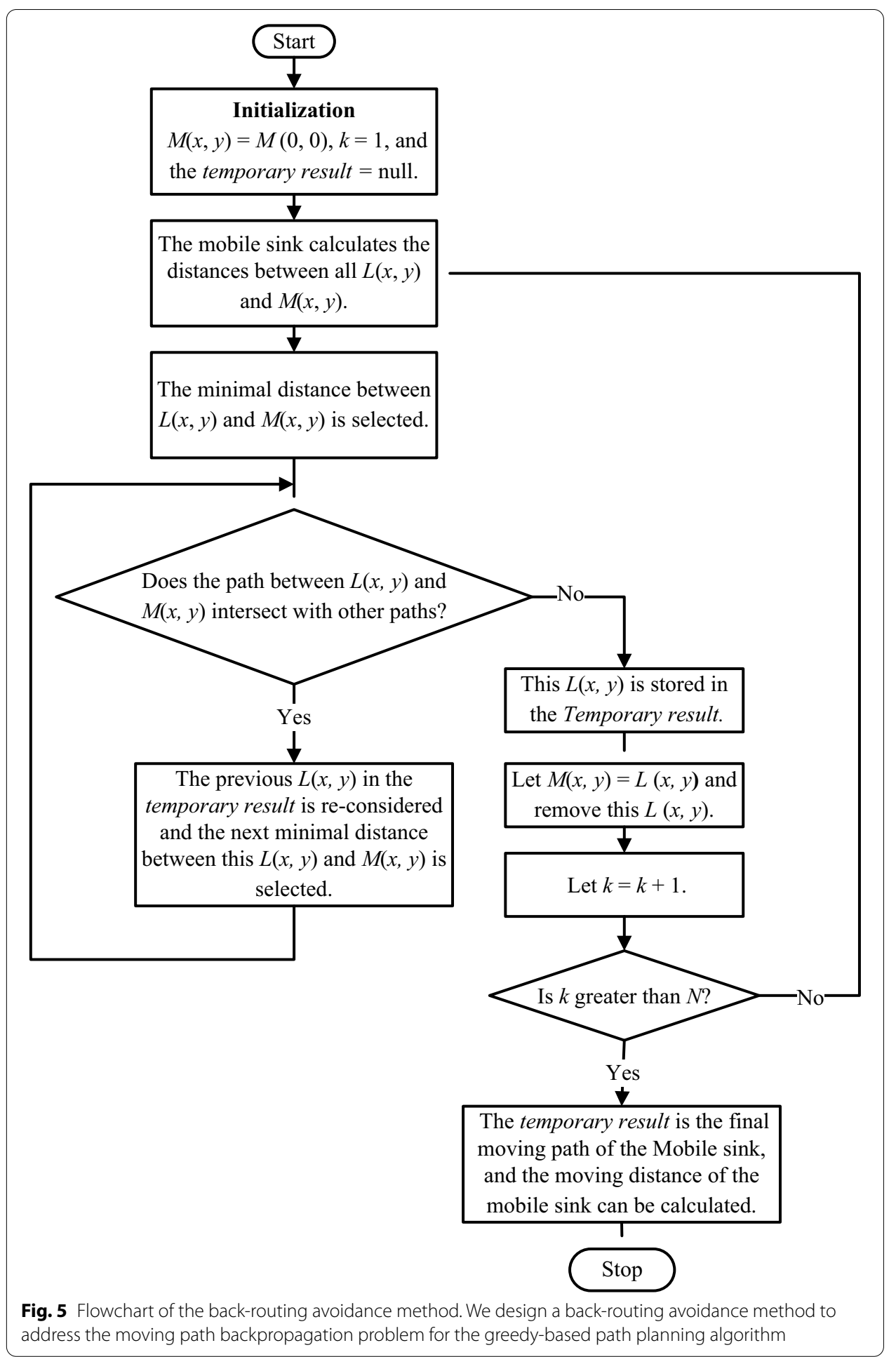

The inner center can be constructed as the intersection of angle bisectors. It is also the interior point for which the distances to the sides of the triangle are equal, and the angle bisectors meet at the inner center [34-36]. Hence, we adopt an angle bisector concept to create the moving path for the mobile sink. Figure 6 shows an example of a moving path 


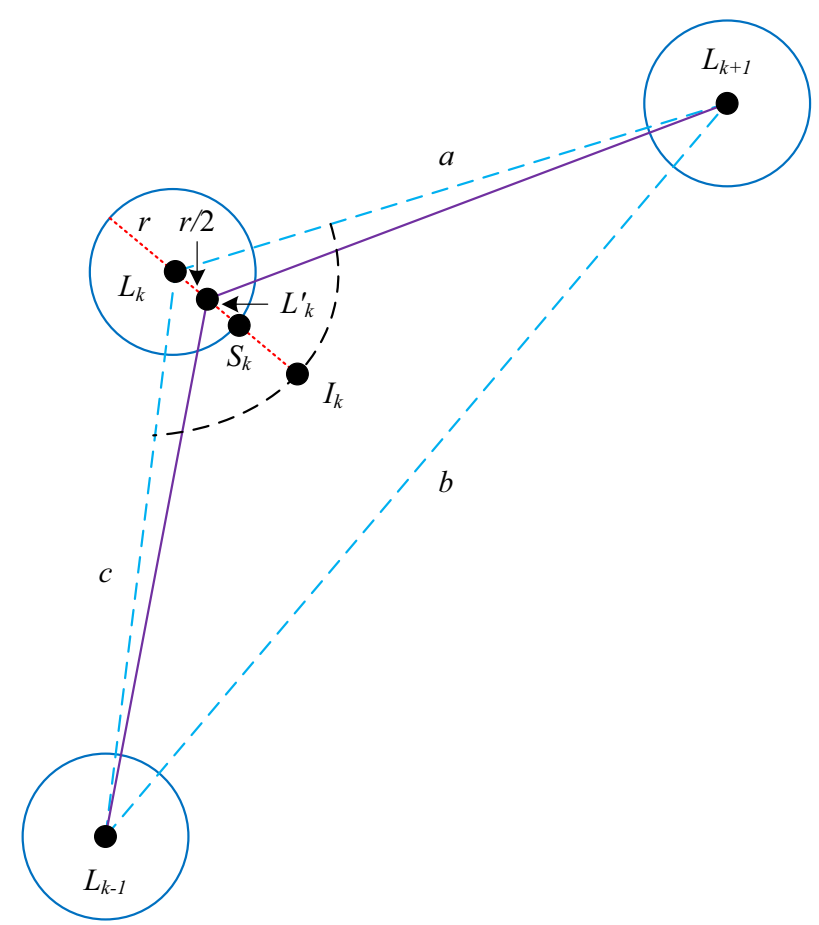

Fig. 6 Example of the moving path calculation for the ICPPA. $L_{k}$ is the location of sensor node $k_{\prime \prime}$ and $r$ is the radius of the communication range for of sensor node. Let $I_{k}\left(I_{k_{x}}, I_{k_{y}}\right)$ be the inner center of sensor node $k$

calculation for the ICPPA, where $L_{k}$ is the location of sensor node $k$ and $r$ is the radius of the communication range of the sensor node. Let $I_{k}\left(I_{k_{x}}, I_{k_{y}}\right)$ be the inner center for sensor node $k$. According to the distances among $L_{k-1}, L_{k}$ and $L_{k+1}, I_{k_{x}}$ and $I_{k_{y}}$ are calculated by [34-36]

$$
\begin{aligned}
I_{k_{x}} & =\frac{a \times L_{k-1_{x}}+b \times L_{k_{x}}+c \times L_{k+1_{x}}}{a+b+c}, \\
I_{k_{y}} & =\frac{a \times L_{k-1_{y}}+b \times L_{k_{y}}+c \times L_{k+1_{y}}}{a+b+c},
\end{aligned}
$$

where $a$ is the distance between $L_{k}$ and $L_{k+1}, b$ is the distance between $L_{k+1}$ and $L_{k-1}$, and $c$ is the distance between $L_{k-1}$ and $L_{k}$. According to the circle center at $L_{k}\left(L_{k_{x}}, L_{k_{y}}\right)$ and the radius $r$, the equation of the circle can be calculated by

$$
\left(x-L_{k_{x}}\right)^{2}+\left(y-L_{k_{y}}\right)^{2}=r^{2} .
$$

According to the path from $I_{k}$ to $L_{k}$, the equation of the line can be calculated by

$$
y-I_{k_{y}}=\frac{L_{k_{y}}-I_{k_{y}}}{L_{k_{x}}-I_{k_{x}}}\left(x-I_{k_{x}}\right) .
$$


By using Eqs. (3) and (4), we calculate the intersection point $S_{k}\left(S_{k_{x}}, S_{k_{y}}\right)$ to obtain the new location $L_{k}^{\prime}\left(L_{k_{x}}^{\prime}, L_{k_{y}}^{\prime}\right)$ for the moving path of the mobile sink. $L_{k}^{\prime}$ can be calculated by

$$
\begin{aligned}
& L_{k_{x}}^{\prime}=\frac{L_{k_{x}}+S_{k_{x}}}{2}, \\
& L_{k_{y}}^{\prime}=\frac{L_{k_{y}}+S_{k_{y}}}{2},
\end{aligned}
$$

We account for the obstacles in the sensing area. The reference point (RP) of obstacle avoidance is employed to address the obstacle problem. A function named Obstacle Avoidance $(u, v)$ is executed to obtain the RP if there is an obstacle between sensor node $u$ and sensor node $v$. Let $A x+B y+C=0$ be the equation of the line between $u\left(x_{1}, y_{1}\right)$ and $v\left(x_{2}, y_{2}\right)$. $A, B$, and $C$ can be calculated according to the calculation of the line equation as follows:

$$
y-y_{1}=\frac{y_{2}-y_{1}}{x_{2}-x_{1}}\left(x-x_{1}\right)
$$

where $A=y_{2}-y_{1}, B=x_{1}-x_{2}$, and $C=x_{2} y_{1}-x_{1} y_{2}$. Let $C P\left(x_{0}, y_{0}\right)$ be the center point of the obstacle and $P\left(P_{x}, P_{y}\right)$ be the projection point from $C P$ to the moving path between $u\left(x_{1}, y_{1}\right)$ and $v\left(x_{2}, y_{2}\right)$. According to $A x+B y+C=0, P_{x}$ and $P_{y}$ are calculated by

$$
\begin{aligned}
& P_{x}=x_{0}-A \times \frac{A x_{0}+B y_{0}+C}{A^{2}+B^{2}}, \\
& P_{y}=y_{0}-B \times \frac{A x_{0}+B y_{0}+C}{A^{2}+B^{2}},
\end{aligned}
$$

For each obstacle, there are four RPs $(R 1, R 2, R 3$ and $R 4)$ near the corner point of the obstacle. The distance between an RP and an obstacle can be determined according to the size of the mobile device. Based on $P\left(P_{x}, P_{y}\right)$, the RP closest to $P\left(P_{x}, P_{y}\right)$ can be selected. When the minimal distances from $P\left(P_{x}, P_{y}\right)$ to two RPs are the same, these two RPs are selected to avoid the obstacle. Figures 7 and 8 show two examples of moving paths with obstacles, where $C P$ is the center point of the obstacle and $P$ is the projection point from $C P$ to the moving path between $L_{k}$ and $L_{k+1}$. According to $P, R 4$ is selected to avoid the obstacle in Fig. 7. Figure 8 shows that $R 2$ and $R 3$ are selected to avoid the obstacle in this case. In the proposed scheme, we design a back-routing avoidance method to address the moving path backpropagation problem faced by the CCPPA. Based on the CCPPA, the proposed scheme includes the back-routing avoidance method and a function for obstacle avoidance. Figure 9 shows the flowchart of the inner center path planning algorithm, where the temporary result of the initialization process is obtained from the back-routing avoidance method, $N$ is the number of sensor nodes, $S_{k}(x, y)$ is the intersection point, and $L_{k}^{\prime}(x, y)$ is the new location of the mobile sink. 


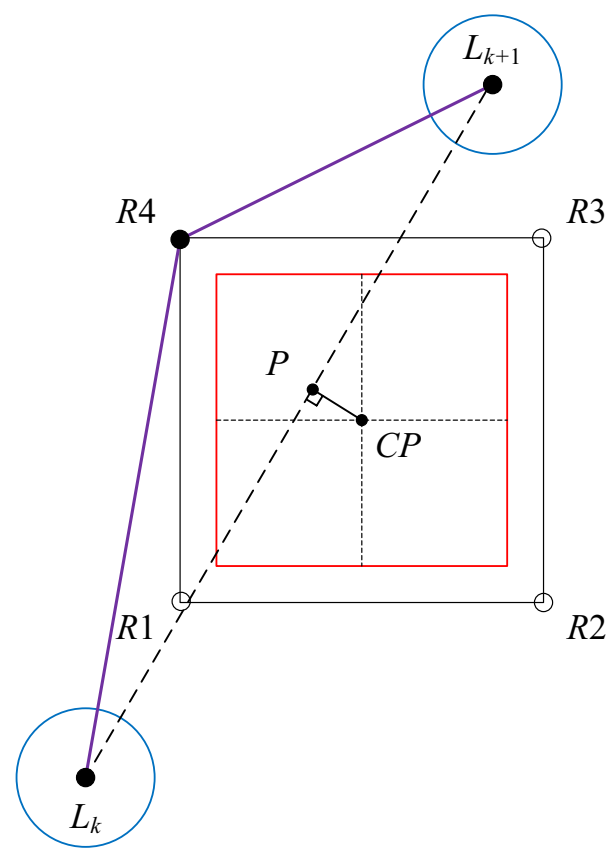

Fig. 7 Example of a moving path with an obstacle (one RP). $C P$ is the center point of the obstacle, and $P$ is the projection point from $C P$ to the moving path between $L_{k}$ and $L_{k+1}$. According to $P, R 4$ is selected to avoid the obstacle in Fig. 7

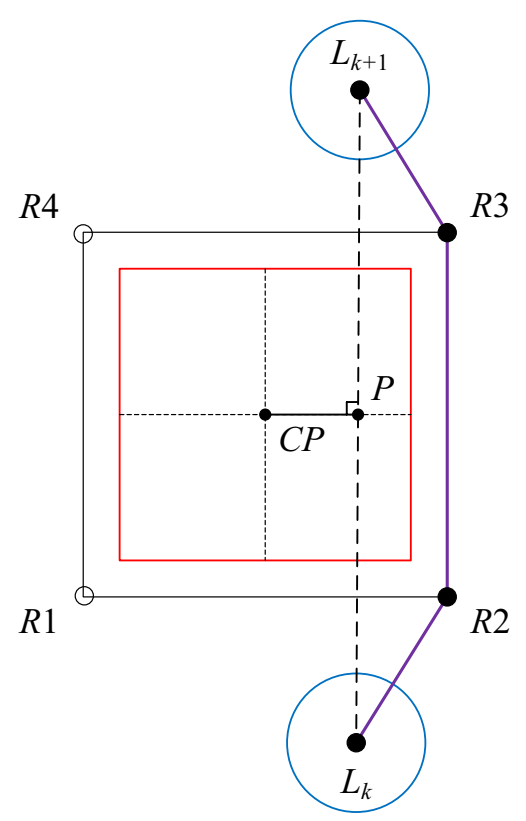

Fig. 8 Example of a moving path with an obstacle (two RPs). CP is the center point of the obstacle, and $P$ is the projection point from $C P$ to the moving path between $L_{k}$ and $L_{k+1}$. According to $P, R 2$ and $R 3$ are selected to avoid the obstacle in this case 


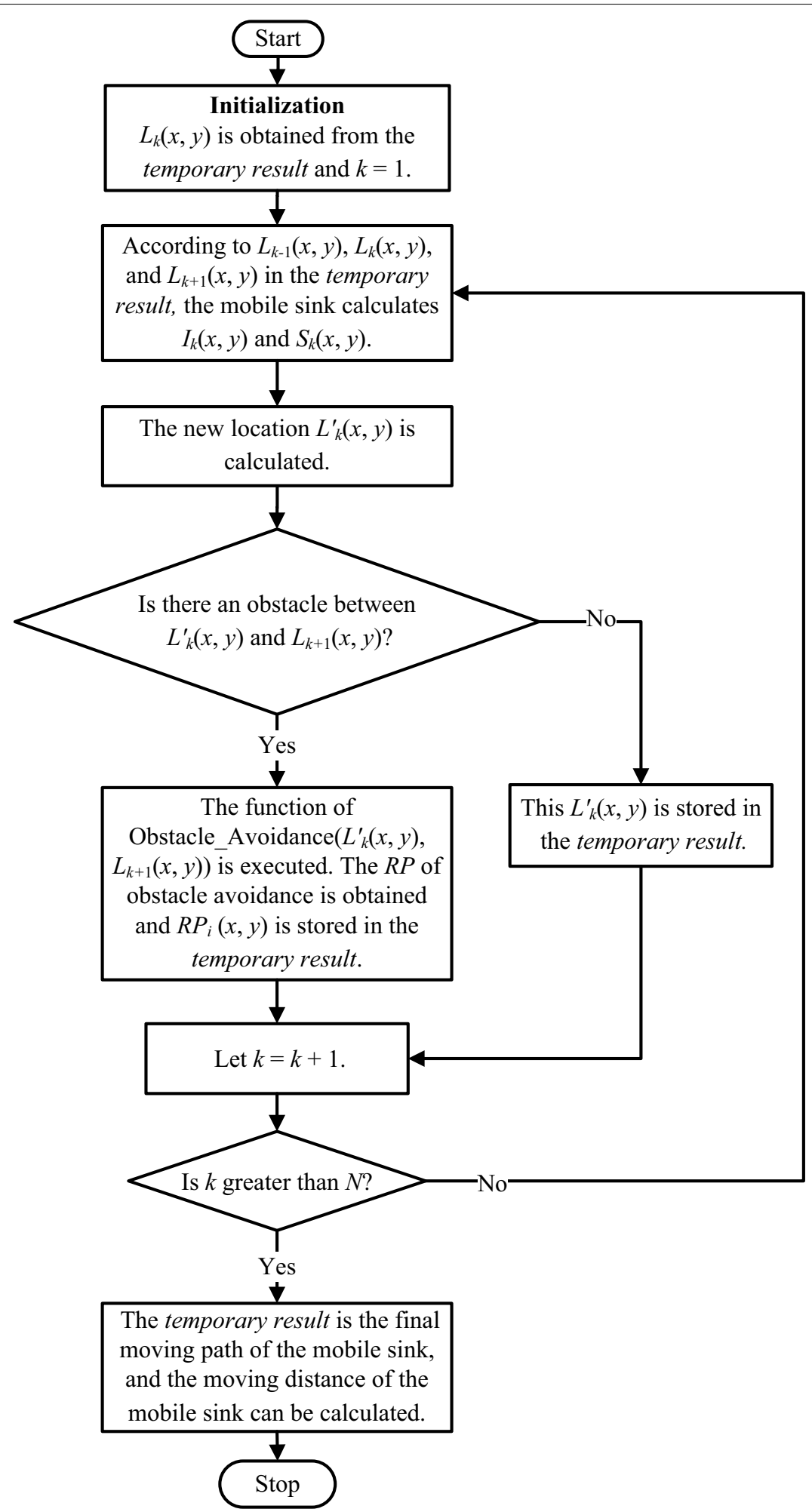

Fig. 9 Flowchart of the inner center path planning algorithm. We design a back-routing avoidance method to address the moving path backpropagation problem faced by the CCPPA. Based on the CCPPA, the proposed scheme includes the back-routing avoidance method and a function for obstacle avoidance 


\section{Results and discussion}

In this section, we evaluate the performance of our proposed scheme by using a simulation model. We describe our simulation environment and illustrate the simulation results, and we compare our scheme with several other schemes. To prove that our proposed scheme is promising, we design a visual interface simulator by using MATLAB and $\mathrm{C \#}$ and implement several other schemes for fair comparison. The assumptions for our simulation environment and the specifications of our data collection process are as follows:

- The simulation environment is composed of a mobile sink, some fixed sensor nodes and several obstacles.

- The radius of the communication range of each sensor node is $50 \mathrm{~m}$, each obstacle in the sensing area is a large square building, and the length of a side of each obstacle is $50 \mathrm{~m}$.

- The sink can randomly move within the sensing area to collect the sensing data.

- The location of each sensor node is randomly distributed in the sensing area.

- Each of the sensor nodes can send data to the mobile sink within its communication range.

- All of the sensor nodes in the network are homogeneous, and the energy of each sensor node can be replenished.

- All of the sensor nodes are stationary, and the initial energy is the same for each sensor node.

- The sensor nodes can continually sense and transfer data.

To prove that our proposed scheme is well designed, the moving paths for the different schemes are analyzed in detail by using the visual interface simulator. We illustrate a snapshot of the moving path for a certain iteration of the simulation by evaluating the CCPPA and our proposed ICPPA, where the sensing area is $1000 \mathrm{~m} \times 1000 \mathrm{~m}$ and the top left corner is the starting position for the mobile sink to travel through all of the sensor nodes. The mobile sink must travel through all of the sensor nodes and then move back to the starting position.

We use the same proposed function for back-routing avoidance with the CCPPA. Figure 10 shows an example of a moving path obtained by using the CCPPA, where the number of sensor nodes is equal to 10 and there are no obstacles. The moving distance of the mobile sink is $3028 \mathrm{~m}$. Figure 11 shows an example of a moving path obtained by using the ICPPA, where the moving distance of the mobile sink is $2829 \mathrm{~m}$. In Fig. 4, the moving distance of the mobile sink is $3413 \mathrm{~m}$, where the CCPPA results in the moving path backpropagation problem. Simulation results show that our proposed function for back-routing avoidance can efficiently address the moving path backpropagation problem.

We also use the same proposed function for obstacle avoidance with the CCPPA. Figure 12 shows an example of a moving path obtained by using the CCPPA when obstacles are present, where the number of sensor nodes is equal to 10 and the number of obstacles is equal to 5 . The moving distance of the mobile sink is $3038 \mathrm{~m}$. Figure 13 shows an example of a moving path obtained by using the ICPPA when obstacles are 


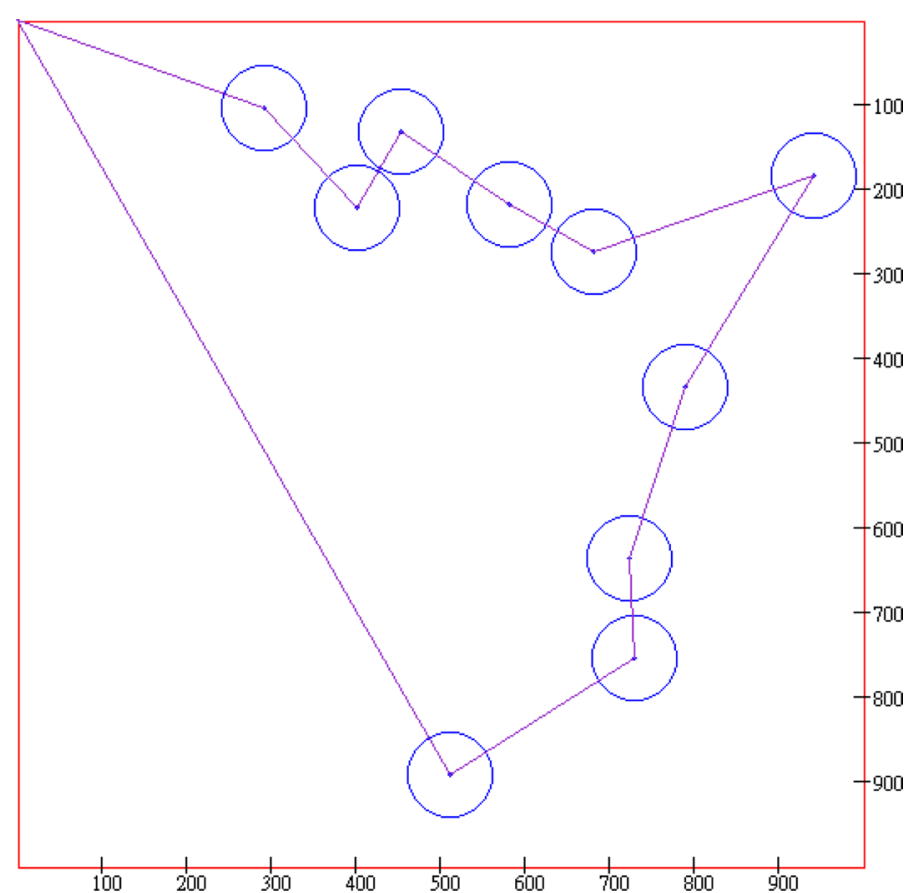

Fig. 10 Moving path for the CCPPA $(1000 \mathrm{~m} \times 1000 \mathrm{~m})$. We use the same proposed function for back-routing avoidance with the CCPPA. This figure shows an example of a moving path obtained by using the CCPPA, where the number of sensor nodes is equal to 10 and there are no obstacles. The moving distance of the mobile sink is $3028 \mathrm{~m}$

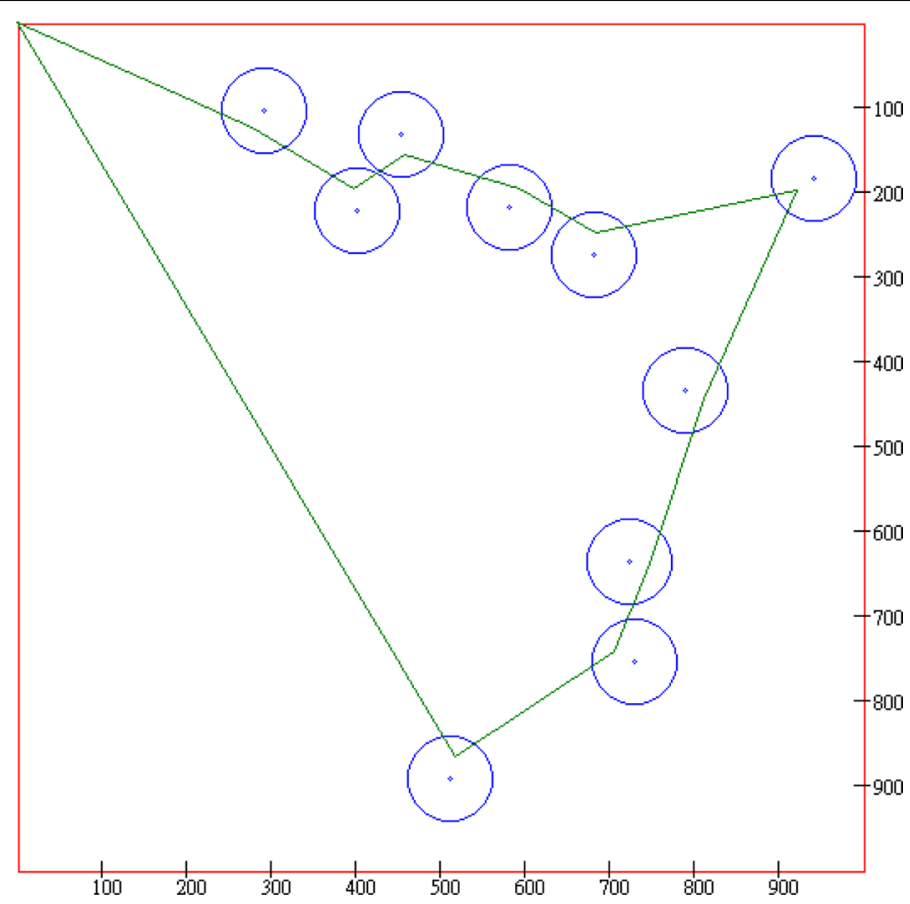

Fig. 11 Moving path for the ICPPA $(1000 \mathrm{~m} \times 1000 \mathrm{~m})$. This figure shows an example of a moving path obtained by using the ICPPA, where the number of sensor nodes is equal to 10 and there are no obstacles. The moving distance of the mobile sink is $2829 \mathrm{~m}$ 


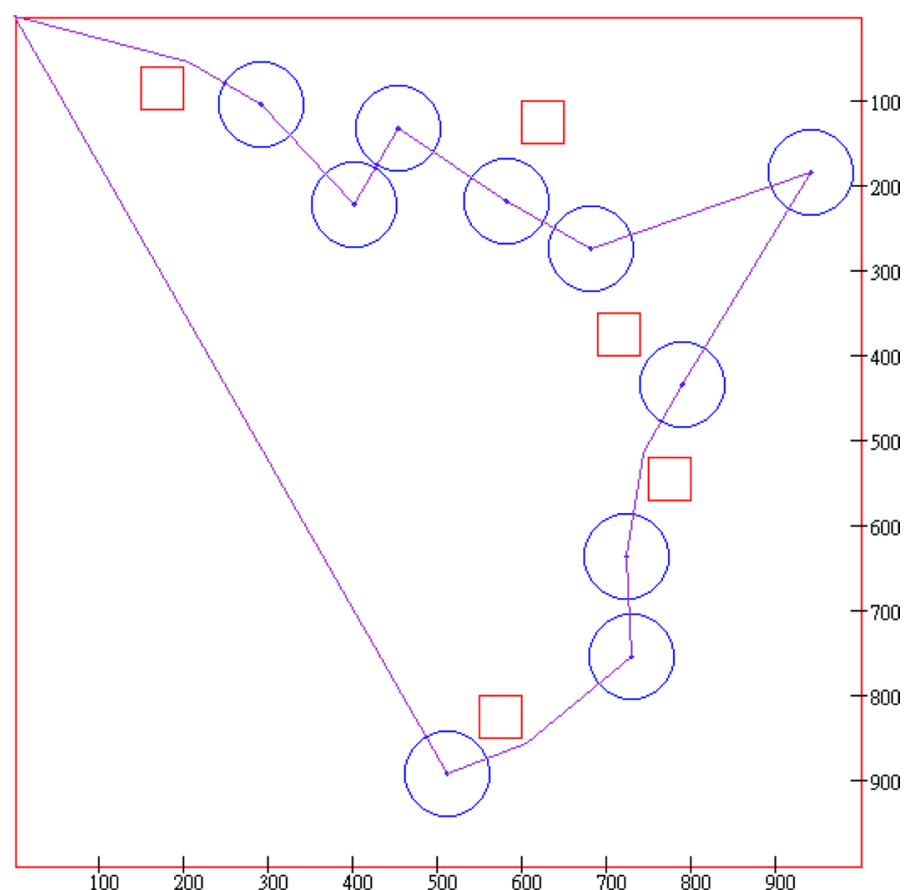

Fig. 12 Moving path for the CCPPA when obstacles are present $(1000 \mathrm{~m} \times 1000 \mathrm{~m})$. This figure shows an example of a moving path obtained by using the CCPPA when obstacles are present, where the number of sensor nodes is equal to 10 and the number of obstacles is equal to 5 . The moving distance of the mobile sink is $3038 \mathrm{~m}$

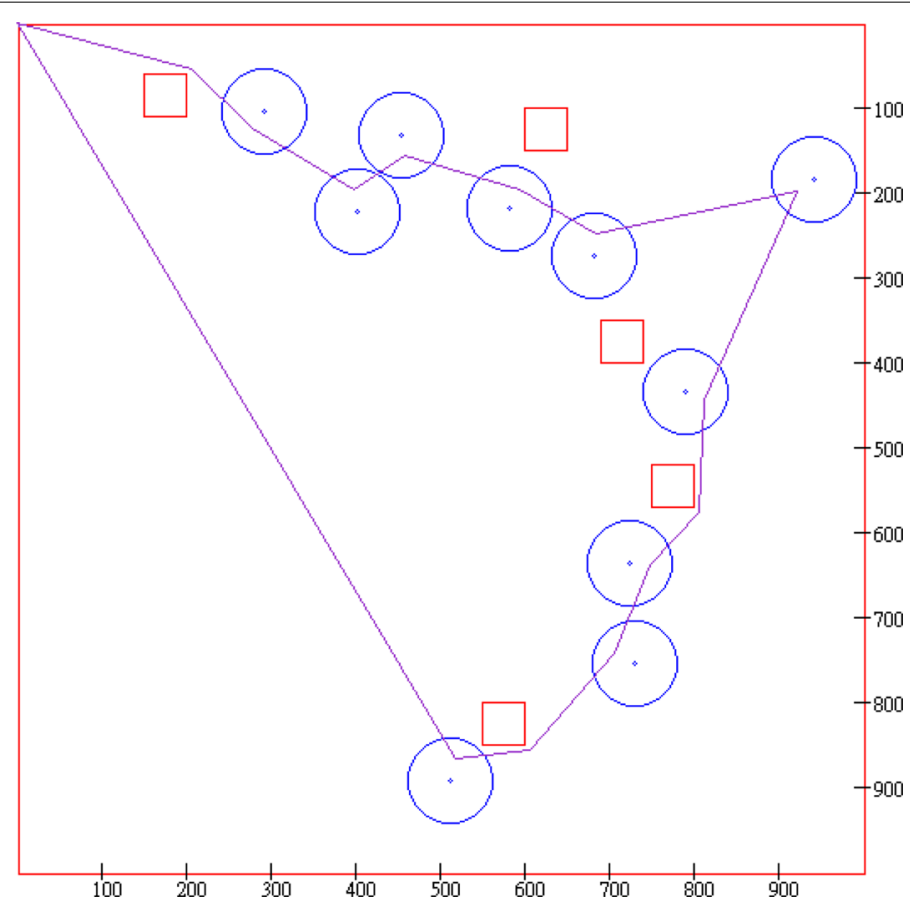

Fig. 13 Moving path for the ICPPA when obstacles are present $(1000 \mathrm{~m} \times 1000 \mathrm{~m})$. This figure shows an example of a moving path obtained by using the ICPPA when obstacles are present, where the number of sensor nodes is equal to 10 and the number of obstacles is equal to 5 . The moving distance of the mobile sink is $2862 \mathrm{~m}$ 


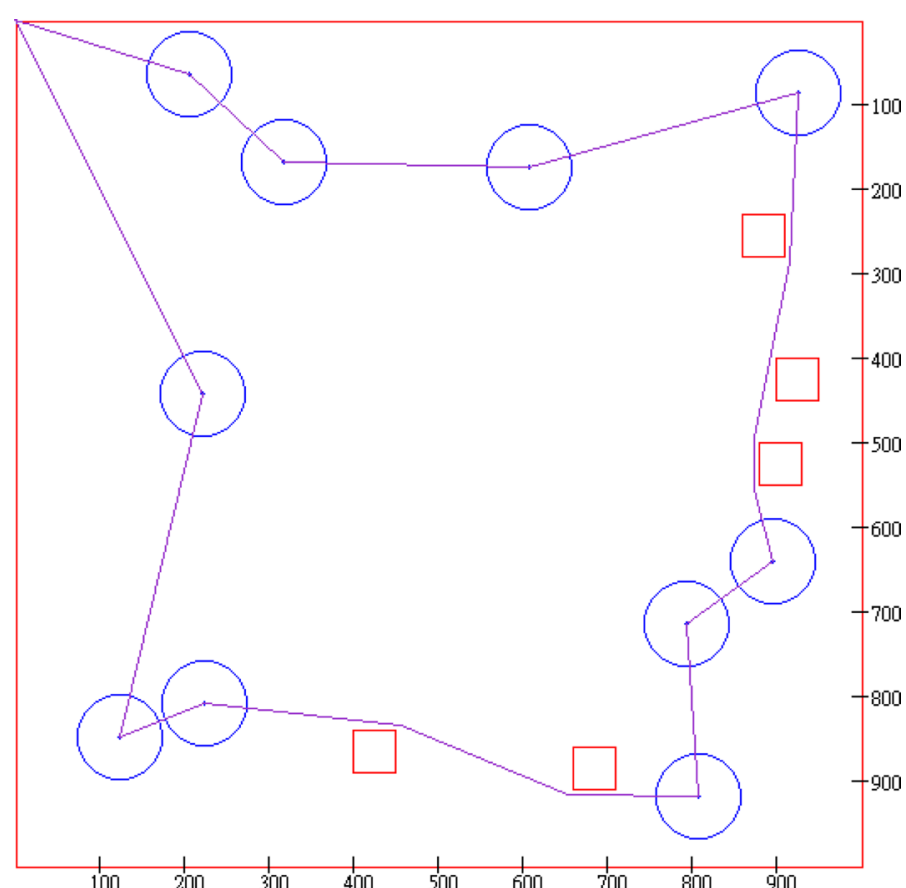

Fig. 14 Moving path for the CCPPA when continuous obstacles are present $(1000 \mathrm{~m} \times 1000 \mathrm{~m})$. This figure shows an example of a moving path obtained by using the CCPPA when continuous obstacles are present, where the moving distance of the mobile sink is $3502 \mathrm{~m}$

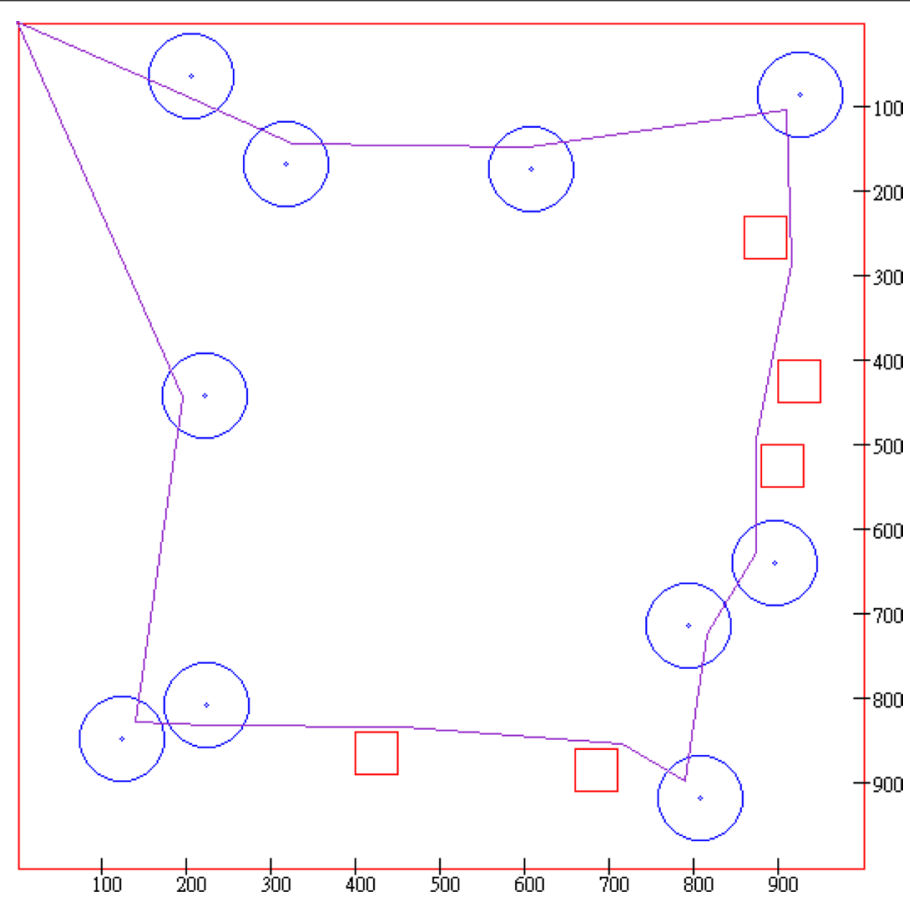

Fig. 15 Moving path for the ICPPA when continuous obstacles are present $(1000 \mathrm{~m} \times 1000 \mathrm{~m})$. This figure shows an example of a moving path obtained by using the ICPPA when continuous obstacles are present, where the moving distance of the mobile sink is $3296 \mathrm{~m}$ 
Table 1 Comparison of five schemes in terms of their moving distances (sensing area: $1000 \mathrm{~m} \times 1000 \mathrm{~m}$ )

\begin{tabular}{llllll}
\hline Scheme & Node $=\mathbf{1 0}$ & Node $=\mathbf{2 0}$ & Node $=\mathbf{3 0}$ & Node $=\mathbf{4 0}$ & Node $=\mathbf{5 0}$ \\
\hline CCPPA_B & 3338.775 & 4263.916 & 5036.789 & 5690.48 & 6269.015 \\
ICPPA_B & 3111.391 & 3839.629 & 4431.876 & 4922.581 & 5346.46 \\
CCPPA_A & 3427.487 & 4462.973 & 5325.835 & 5988.507 & 6617.562 \\
ICPPA_A & 3194.238 & 4036.936 & 4722.342 & 5228.729 & 5715.285 \\
HTPA & 3257.01 & 4145.382 & 4869.18 & 5411.259 & 5924.781 \\
\hline
\end{tabular}

Table 2 Comparison of five schemes in terms of their moving distances (sensing area: $2000 \mathrm{~m} \times 2000 \mathrm{~m}$ )

\begin{tabular}{lllcll}
\hline Scheme & Node $=\mathbf{1 0}$ & Node $=\mathbf{2 0}$ & Node $=\mathbf{3 0}$ & Node $=\mathbf{4 0}$ & Node $=\mathbf{5 0}$ \\
\hline CCPPA_B & 6930.978 & 9044.038 & $10,663.25$ & $12,023.8$ & $13,201.87$ \\
ICPPA_B & 6684.225 & 8576.513 & 9981.743 & $11,142.05$ & $12,123.07$ \\
CCPPA_A & 7124.837 & 9451.558 & $11,198.09$ & $12,626.94$ & $13,905.48$ \\
ICPPA_A & 6873.285 & 8981.423 & $10,521.83$ & $11,755.29$ & $12,848.36$ \\
HTPA & 6948.583 & 9120.269 & $10,718.83$ & $12,004.62$ & $13,147.07$ \\
\hline
\end{tabular}

present, where the moving distance of the mobile sink is $2862 \mathrm{~m}$. To prove that our proposed function for obstacle avoidance is well designed, we account for an environment with continuous obstacles. Figure 14 shows an example of a moving path obtained by using the CCPPA when continuous obstacles are present, where the moving distance of the mobile sink is $3502 \mathrm{~m}$. Figure 15 shows an example of a moving path obtained by using the ICPPA when continuous obstacles are present, where the moving distance of the mobile sink is $3296 \mathrm{~m}$. From the simulation results, it is evident that the CCPPA results in a longer moving distance of the mobile sink than that yielded by the ICPPA. In accordance with Figs. 10, 11, 12, 13, 14 and 15, the moving paths illustrate that the proposed scheme leads to a shorter moving distance of the mobile sink in the sensing area with different locations of sensor nodes and obstacles. The proposed scheme makes an adaptive decision for creating the moving path of the mobile sink. The moving distance of the mobile sink is shortened by using the function for back-routing avoidance and the function for obstacle avoidance. Hence, reasonable moving path planning can be achieved, and the moving distance of the mobile sink can be reduced.

The performance measures obtained on the basis of one hundred simulation runs are plotted as a function of the moving distance. For each simulation run, different locations of the sensor nodes are created based on the same number of sensor nodes. To prove that our proposed scheme is well designed, we evaluate the performance of the CCPPA_A, ICPPA_A, CCPPA_B, ICPPA_B and HTPA [21]. The back-routing avoidance method is not included in the CCPPA_A and ICPPA_A. In contrast, the CCPPA_B and ICPPA_B execute the function for back-routing avoidance. Tables 1 and 2 show the comparison of five schemes in terms of their moving distances when the sensing areas are $1000 \mathrm{~m} \times 1000 \mathrm{~m}$ and $2000 \mathrm{~m} \times 2000 \mathrm{~m}$, where the numbers of sensor nodes are 10, 20, 30, 40 and 50. Table 3 shows the comparison of five schemes in terms of their moving distances when the sensing area is $5000 \mathrm{~m} \times 5000 \mathrm{~m}$ and the numbers of sensor nodes 
Table 3 Comparison of five schemes in terms of their moving distances (sensing area: $5000 \mathrm{~m} \times \mathbf{5 0 0 0} \mathrm{m}$ )

\begin{tabular}{llllll}
\hline Scheme & Node $=\mathbf{5 0}$ & Node $=\mathbf{1 0 0}$ & Node $=\mathbf{1 5 0}$ & Node $=\mathbf{2 0 0}$ & Node $=\mathbf{2 5 0}$ \\
\hline CCPPA_B & 6930.978 & 9044.038 & $10,663.25$ & $12,023.8$ & $13,201.87$ \\
ICPPA_B & 6684.225 & 8576.513 & 9981.743 & $11,142.05$ & $12,123.07$ \\
CCPPA_A & 7124.837 & 9451.558 & $11,198.09$ & $12,626.94$ & $13,905.48$ \\
ICPPA_A & 6873.285 & 8981.423 & $10,521.83$ & $11,755.29$ & $12,848.36$ \\
HTPA & 6948.583 & 9120.269 & $10,718.83$ & $12,004.62$ & $13,147.07$ \\
\hline
\end{tabular}

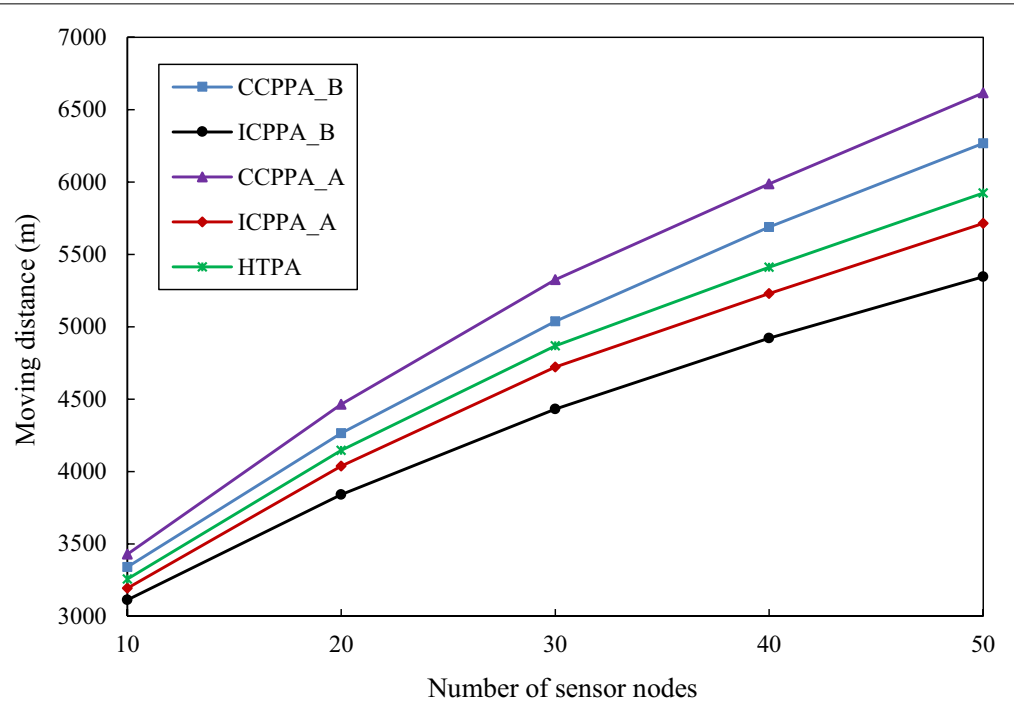

Fig. 16 Moving distances $(1000 \mathrm{~m} \times 1000 \mathrm{~m})$. This figure shows the moving distances yielded when the sensing area is $1000 \mathrm{~m} \times 1000 \mathrm{~m}$, where the numbers of sensor nodes are 10,20,30, 40 and 50 . We evaluate the performances of five schemes. The back-routing avoidance method is not included in the CCPPA_A and ICPPA_A. In contrast, the CCPPA_B and ICPPA_B execute the function for back-routing avoidance

are 50,100, 150, 200 and 250. According to the three tables, it is clear that the ICPPA_B results in a shorter moving distance for the mobile sink than the other algorithm/function combinations. In Table 1, we can observe the difference between the ICPPA_B and the HTPA. When the number of sensor nodes in the sensing area is increased by 10 , the ICPPA_B yields an average moving distance that is $108 \mathrm{~m}$ shorter than that of the HTPA. In Table 2, when the number of sensor nodes in the sensing area is increased by 10, the ICPPA_B yields an average moving distance that is $190 \mathrm{~m}$ shorter than that of the HTPA. In Table 3, when the number of sensor nodes in the sensing area is increased by 50, the ICPPA_B yields an average moving distance that is $1.1 \mathrm{~km}$ shorter than that of the HTPA.

Figures 16 and 17 show the moving distances obtained by the algorithms when the sensing areas are $1000 \mathrm{~m} \times 1000 \mathrm{~m}$ and $2000 \mathrm{~m} \times 2000 \mathrm{~m}$, where the numbers of sensor nodes are 10, 20,30, 40 and 50, respectively. The moving distances of the five schemes exhibit similar trends when the number of sensor nodes varies from 10 to 50. Clearly, the moving distance of the CCPPA_A is longer than that of the CCPPA_B, and the moving distance of the ICPPA_A is longer than that of the ICPPA_B. For 


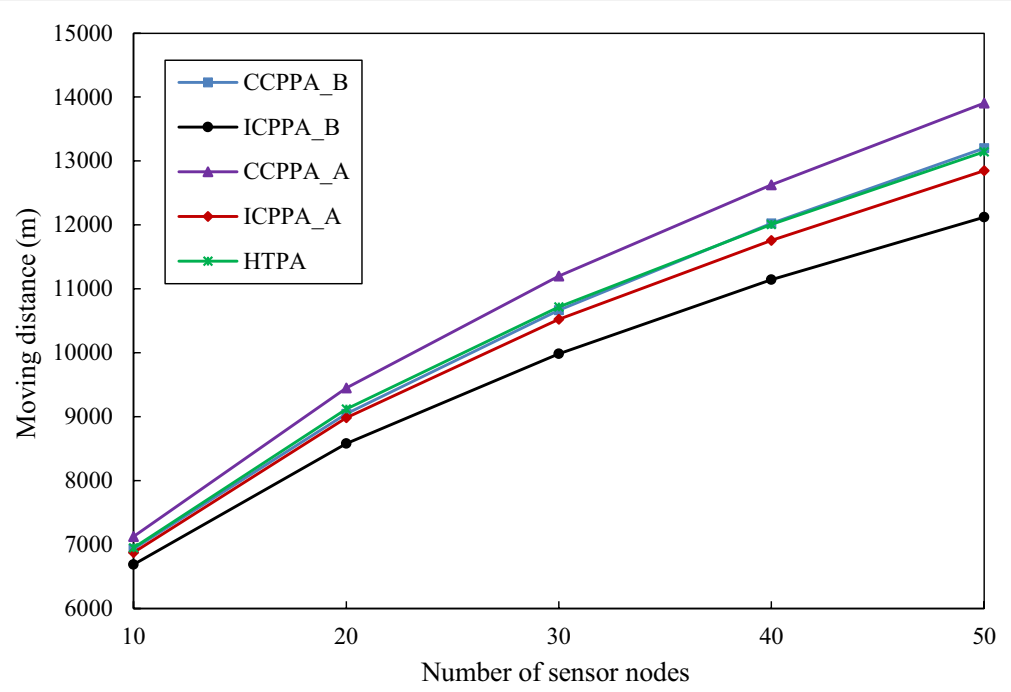

Fig. 17 Moving distances $(2000 \mathrm{~m} \times 2000 \mathrm{~m})$. This figure shows the moving distances yielded when the sensing area is $2000 \mathrm{~m} \times 2000 \mathrm{~m}$, where the numbers of sensor nodes are 10, 20,30, 40 and 50 . We evaluate the performances of five schemes. The back-routing avoidance method is not included in the CCPPA_A and ICPPA_A. In contrast, the CCPPA_B and ICPPA_B execute the function for back-routing avoidance

example, when the number of sensor nodes is 50 in Fig. 16, the ICPPA_B easily achieves a reduction in the moving distance of the mobile sink from 6617 to $5346 \mathrm{~m}$ when compared with that of the CCPPA_A. Furthermore, in this case, the ICPPA_B achieves a moving distance of the mobile sink that is approximately $923 \mathrm{~m}$ shorter than that of the CCPPA_B. Because the sensing area is extended, the mobile sink must move a long distance when employing the CCPPA, and this results in significant energy consumption by the mobile sink. The HTPA belongs to the class of shortest path prioritizing methods. The moving distance for the mobile sink is close to that of the CCPPA_B. The proposed ICPPA_A and ICPPA_B result in shorter moving distances when the number of sensor nodes increases. The reason for this behavior is that we account for the communication range limitations of sensor nodes and make an efficient decision for creating the moving path of the mobile sink. The moving path is dynamically adaptive based on the positions of the sensor nodes. Hence, the ICPPA_A and ICPPA_B result in an improved moving path for the mobile sink and reduce the moving distances of the mobile sink. This implies that the energy consumption of the mobile sink can be reduced in WSNs. Moreover, to prove that our proposed scheme is promising in large-scale WSNs, we extend the sensing area in the simulation environment. Figure 18 shows the moving distances when the sensing area is $5000 \mathrm{~m} \times 5000 \mathrm{~m}$ and the numbers of sensor nodes are 50,100, 150, 200 and 250. The CCPPA_A and ICPPA_A do not perform the function of back-routing avoidance, which causes the moving path backpropagation problem. Hence, the moving distances increase noticeably when the number of sensor nodes increases.

In accordance with Figs. 16, 17 and 18, the curves illustrate that the proposed scheme leads to shorter moving distances in different sensing areas. The proposed scheme provides a suitable moving path of the mobile sink. This finding occurs because we adopt an 


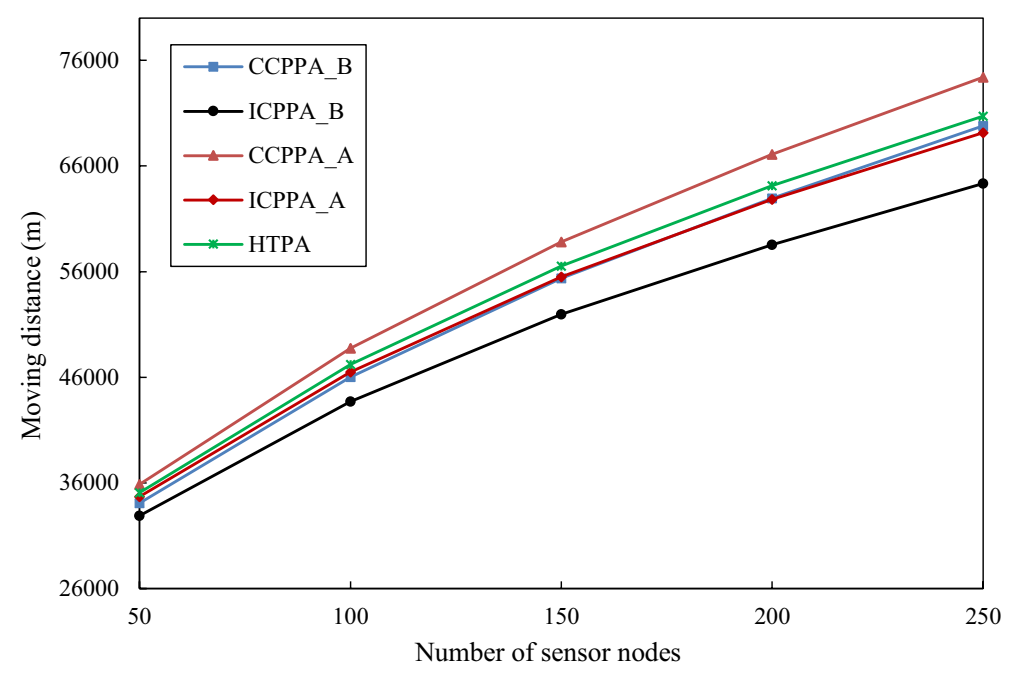

Fig. 18 Moving distances $(5000 \mathrm{~m} \times 5000 \mathrm{~m})$. This figure shows the moving distances when the sensing area is $5000 \mathrm{~m} \times 5000 \mathrm{~m}$ and the numbers of sensor nodes are 50, 100, 150, 200 and 250 . We evaluate the performances of five schemes. The back-routing avoidance method is not included in the CCPPA_A and ICPPA_A. In contrast, the CCPPA_B and ICPPA_B execute the function for back-routing avoidance

angle bisector concept to create the moving path for the mobile sink. Efficient moving path planning for a mobile sink can be achieved.

In view of Figs. 10, 11, 12, 13, 14, 15, 16, 17 and 18, it is clear that a suitable moving distance for the mobile sink is an important feature in the issues of energy consumption. Our proposed scheme shortens the moving distance of the mobile sink. Because of the better moving path planning in the proposed scheme, it is intuitive that the proposed scheme results in a shorter distance than that of the other schemes in a large-scale sensing area. This implies that the lifetime of the mobile sink can be extended to perform more data collection tasks.

\section{Conclusion}

The development of a path planning scheme is a challenging issue in wireless sensor networks with mobile sinks. In this paper, an efficient path planning scheme employing the back-routing avoidance method and a function for obstacle avoidance is proposed to reduce the moving distance so that a significant improvement in the energy savings and lifetimes of mobile sinks can be achieved in wireless sensor networks. In the proposed scheme, we make an adaptive decision for creating the moving path of the mobile sink. The moving path backpropagation problem and obstacle avoidance challenge are solved. The proposed scheme is promising in large-scale wireless sensor networks. When the number of sensor nodes in the sensing area is increased by 50, the proposed scheme yields an average moving distance that is $1.1 \mathrm{~km}$ shorter than that of the heuristic tourplanning algorithm, where the sensing area is $5 \mathrm{~km} \times 5 \mathrm{~km}$. Simulation results indicate that our proposed algorithm achieves suitable moving path planning scheme and a short moving distance for the mobile sink in a wireless sensor network. 


\section{Abbreviations}

WSNs: Wireless sensor networks; BS: Base station; CHs: Cluster heads; LEACH: Low-energy adaptive clustering hierarchy: PE-LEACH: Partitioned-based energy-efficient LEACH; CCPPA: Center of circle path planning algorithm; ICPPA: Inner center path planning algorithm; RP: Reference point; CP: Center point of an obstacle.

\section{Authors' contributions}

Jau-Yang Chang conceived this study and wrote the entire article. Jin-Tsong Jeng and Yung-Hoh Sheu verified the analytical methods. Z-Jie Jian and Wei-Yeh Chang carried out the simulations and prepared the graphs. All authors read and approved the final manuscript.

\section{Funding}

This work was supported by the Ministry of Science and Technology, Taiwan, under Grant MOST 108-2221-E-150-043.

\section{Availability of data and materials}

The data sets used and/or analyzed during the current study are available from the corresponding author upon reasonable request.

\section{Competing interests}

The authors declare that they have no conflicts of interest.

Received: 15 June 2020 Accepted: 2 December 2020

Published online: 14 December 2020

\section{References}

1. W.Z. Khan, M.H. Rehman, H.M. Zangoti, M.K. Afzal, N. Armi, K. Salah, Industrial internet of things: Recent advances, enabling technologies and open challenges. Comput. Electr. Eng. 81, 1-13 (2020)

2. N.A. Pantazis, S.A. Nikolidakis, D.D. Vergados, Energy-efficient routing protocols in wireless sensor networks: a survey. IEEE Commu Surv Tutor 15(2), 551-591 (2013)

3. M.H. Khodashahi, F. Tashtarian, M.H.Y. Mohammad, M.T. Honary, in Proceedings IEEE Wireless Communications and Networking Conference (WCNC), Optimal Location for Mobile Sink in Wireless Sensor Networks (2010), pp. 1-6.

4. C.F. Wang, J.D. Shih, B.H. Pan, T.Y. Wu, A network lifetime enhancement method for sink relocation and its analysis in wireless sensor networks. IEEE Sens. J. 14(6), 1932-1943 (2014)

5. H. Salarian, K.-W. Chin, F. Naghdy, An energy-efficient mobile-sink path selection strategy for wireless sensor networks. IEEE Trans. Veh. Technol. 63(5), 2407-2419 (2014)

6. C. Tunca, S. Isik, M.Y. Donmez, C. Ersoy, "Ring routing: an energy-efficient routing protocol for wireless sensor networks with a mobile sink. IEEE Trans. Mob. Comput. 14(9), 1947-1960 (2015)

7. M. Abo-Zahhad, S.M. Ahmed, N. Sabor, S. Sasaki, Mobile sink-based adaptive immune energy-efficient clustering protocol for improving the lifetime and stability period of wireless sensor networks. IEEE Sens. J. 15(8), 4576-4586 (2015)

8. J.-Y. Chang, T.-H. Shen, An efficient tree-based power saving scheme for wireless sensor networks with mobile sink. IEEE Sens. J. 16(20), 7545-7557 (2016)

9. T.A. Al-Janabi, H.S. Al-Raweshidy, A centralized routing protocol with a scheduled mobile sink-based Al for large scale I-IoT. IEEE Sens. J. 18(24), 10248-10261 (2018)

10. N. Gharaei, K. Abu Bakar, S.Z.M. Hashim, A.H. Pourasl, Energy-efficient intra-cluster routing algorithm to enhance the coverage time of wireless sensor networks. IEEE Sens J. 19(12), 4501-4508 (2019)

11. W. Wen, S. Zhao, C. Shang, C.-Y. Chang, EAPC: energy-aware path construction for data collection using mobile sink in wireless sensor networks. IEEE Sens. J. 18(2), 890-901 (2018)

12. C.-F. Cheng, C.-F. Yu, Mobile data gathering with bounded relay in wireless sensor networks. IEEE Int. Things J. 5(5), 3891-3907 (2018)

13. X. Gu, X. Zhou, B. Yua, Y. Sun, A Bayesian compressive data gathering scheme in wireless sensor networks with one mobile sink. IEEE Access 6, 47897-47910 (2018)

14. R. Deng, S. He, J. Chen, An online algorithm for data collection by multiple sinks in wireless-sensor networks. IEEE Trans. Control Netw. Syst. 5(1), 93-104 (2018)

15. K.L.-M. Ang, J.K. Phooi Seng, A.M. Zungeru, Optimizing energy consumption for big data collection in large-scale wireless sensor networks with mobile collectors. IEEE Syst. J. 12(1), 616-626 (2018)

16. X. He, S. Liu, G. Yang, N. Xiong, Achieving efficient data collection in heterogeneous sensing WSNs. IEEE Access $\mathbf{6}$, 63187-63199 (2018)

17. Y. Zhang, S. He, J. Chen, Near optimal data gathering in rechargeable sensor networks with a mobile sink. IEEE Trans. Mob. Comput. 16(6), 1718-1729 (2017)

18. C. Wu, Y. Liu, F. Wu, W. Fan, B. Tang, Graph-based data gathering scheme in WSNs with a mobility-constrained mobile sink. IEEE Access 5, 19463-19477 (2017)

19. Z. Zhou, C. Du, L. Shu, G. Hancke, J. Niu, H. Ning, An energy-balanced heuristic for mobile sink scheduling in hybrid WSNs. IEEE Trans. Industr. Inf. 12(1), 28-40 (2016)

20. A.W. Khan, A.H. Abdullah, M.A. Razzaque, J.I. Bangash, VGDRA: a virtual grid-based dynamic routes adjustment scheme for mobile sink-based wireless sensor networks. IEEE Sens. J. 15(1), 526-534 (2015)

21. G. Xie, F. Pan, Cluster-based routing for the mobile sink in wireless sensor networks with obstacles. IEEE Access 4 2019-2028 (2016)

22. A. Alomari, W. Phillips, N. Aslam, F. Comeau, Swarm intelligence optimization techniques for obstacle-avoidance mobility-assisted localization in wireless sensor networks. IEEE Access 6, 22368-22385 (2018) 
23. H. Guo, C. Shen, H. Zhang, H. Chen, R. Jia, Simultaneous trajectory planning and tracking using an MPC method for cyber-physical systems: a case study of obstacle avoidance for an intelligent vehicle. IEEE Trans. Industr. Inf. 14(9), 4273-4283 (2018)

24. H. Cheon, B.K. Kim, Online bidirectional trajectory planning for mobile robots in state-time space. IEEE Trans. Industr. Inf. 66(6), 4555-4565 (2019)

25. Y. Xie, Z. Zhang, X. Wu, Z. Shi, Y. Chen, B. Wu, K.A. Mantey, Obstacle avoidance and path planning for multi-joint manipulator in a space robot. IEEE Access 8, 3511-3526 (2020)

26. X. Liu, Y. Li, J. Zhang, J. Zheng, C. Yang, Self-adaptive dynamic obstacle avoidance and path planning for USV under complex maritime environment. IEEE Access 7, 114945-114954 (2019)

27. Z. Chen, Y. Zhang, Y. Zhang, Y. Nie, J. Tang, S. Zhu, A hybrid path planning algorithm for unmanned surface vehicles in complex environment with dynamic obstacles. IEEE Access 7, 126439-126449 (2019)

28. H. Zhang, H. Jin, Z. Liu, Y. Liu, Y. Zhu, J. Zhao, Real-time kinematic control for redundant manipulators in a timevarying environment: multiple-dynamic obstacle avoidance and fast tracking of a moving object. IEEE Trans. Industr. Inf. 16, 28-41 (2020)

29. H. Mohapatra, A.K. Rath, Fault tolerance in WSN through PE-LEACH protocol. IET Wireless Sensor Systems 9(6), 358-365 (2019)

30. W.R. Heinzelman, A. Chandrakasan, H. Balakrishnan, Energy-efficient communication protocol for wireless microsensor networks, in Proceedings 33rd Hawaii international conference on system sciences (2000), pp. 1-10

31. S.K. Singh, P. Kumar, J.P. Singh, A survey on successors of LEACH protocol. IEEE Access 5, 4298-4328 (2017)

32. S.E. Khediri, R.U. Khan, N. Nasri, A. Kachouri, MW-LEACH: low energy adaptive clustering hierarchy approach for WSN. IET Wirel. Sens. Syst. 10(3), 126-129 (2020)

33. L. Zhou, Y. Fang, Q. Wei, Y. Jin, Z. Hu, LEACH-TLC: a strategy of reducing and uniform energy consumption based on target location constraint. IET Wirel. Sens. Syst. 9(6), 347-357 (2019)

34. C. Kimberling, A combinatorial classification of triangle centers on the line at infinity. J. Integer Seq. 22, 1-13 (2019)

35. C. Kimberling, Central points and central lines in the plane of a triangle. Math. Mag. 67, 163-187 (1994)

36. M.D. Zoltowski, T.S. Lee, Bisector angle estimation in a nonsymmetric multipath radar scenario. IEE Proc F-Radar Signal Process. 138(6), 559-570 (1991)

\section{Publisher's Note}

Springer Nature remains neutral with regard to jurisdictional claims in published maps and institutional affiliations.

\section{Submit your manuscript to a SpringerOpen ${ }^{\circ}$ journal and benefit from:}

- Convenient online submission

- Rigorous peer review

- Open access: articles freely available online

- High visibility within the field

- Retaining the copyright to your article

Submit your next manuscript at $\gg$ springeropen.com 